\title{
A SERIES OF THIRTY-ONE CASES OF RETINAL DETACHMENT TREATED BY DIATHERMY
}

\author{
BY
}

\author{
E. F. KING
}

LONDON

THE following is a brief survey of the operations performed for detached retina at Moorfields Eye Hospital during the last three years and an analysis of 31 cases treated by diathermy during my last three months there as 'House Surgeon, from August to October, 1932.

Gonin's method of cautery puncture for detachment was begun at Moorfields in December, 1929, and the results were analysed by Shapland, who showed that 27.5 per cent. of the first 200 cases were cured, i.e., left hospital with a full field and no detachment visible. None of these cases had been subjected to any previous detachment operation, nor was any selection exercised and every case which offered the smallest hope of improvement was treated. I have no figures of relapse but this occurred in a relatively small number of cases and it was not felt that the operation should be discredited on this account. It was, however, appreciated that cautery puncture was a drastic operation, necessarily traumatising the vitreous in cases where it was, frequently, already degenerate, and the scarring produced was often excessive, with the result that, though the original hole was closed, a new one was torn in the neighbourhood. Further, in this operation the electro-cautery must "hit off" the retinal tear with absolute precision, which requires extremely accurate localization of the hole, a procedure beset with many difficulties, particularly when the hole is situated on the top of a balloon detachment and at some distance from the periphery of the fundus. For these reasons the Guist operation of multiple trephining with application of solid caustic potash to the exposed choroid was introduced. This has two manifest advantages over Gonin's operation, first, that the field of operation is outside the vitreous chamber, and secondly, a diffuse field of chemical choroiditis is produced, so planned that the estimated position of the tear, when the retina is flat, will be at its centre; in this way the importance of extreme accuracy in localization of the hole, which must contain, in the final instance, an element of luck, is minimised.

Black showed that in the first 30 cases treated by Guist's method, which had not had a previous cautery puncture, 45 per cent. were cured. Here again no selection was exercised and the operation performed in every case that was not entirely hopeless. It is 
notable that in four of these cases no hole was found, the caustic potash being applied to each quadrant of the globe in which detachment was present. Unfortunately, these encouraging results were not maintained, and in the following 42 cases, in which no operation had been done before, there were only 21.4 per cent. of cures and 23.8 per cent. of cases showing improvement, i.e., enlargement of the field though some detachment was still visible; a large proportion of the latter would, of course, be expected to relapse. There appear to have been three cases cured by this operation which had previously been unsuccessfully treated by cautery puncture.

The advantages of the method of diffuse caustic cauterization of the choroid over that of localized thermal cauterization seemed clear, but it was found that the reaction in the eye was often severe, leading to pronounced vitreous haze and opacities. On three occasions in the series of 72 cases a large, opaque, swollen mass appeared in the fundus after operation, presumably due to thrombosis of a vortex vein, and though this largely subsided in the course of time the function of the eye was virtually lost. Moreover, the technical difficulties of multiple trephining of the sclera, often at a considerable distance from the limbus, are very great, for the risk of perforation of the choroid is always present, after which completion of the operation is almost impossible, and it seems doubtful, on this score alone, if the operation will ever attain widespread popularity.

There appear to be two lines of treatment of detached retina by diathermy current corresponding in principle with the Gonin and Guist operations. Weve of Utrecht, like Gonin, aims at sealing the hole in the retina itself. For localization of the hole he uses a "substitution" method. With the head of the patient immobile in a rest, an ophthalmoscope, carried on a rotating arm, is focused on the hole and clamped. The patient is removed and a frosted glass globe of approximately the same dimensions as the eye in question introduced in the exact position of the eye itself. The beam from the ophthalmoscope can be picked up at points where it enters and leaves the globe, the latter point giving the relative position of the hole in the eye. Two limbs of an adjustable tripod are placed at 3 o'clock and 9 o'clock on the "limbus" of the substituted eye, the third limb rests where the beam from the ophthalmoscope is picked up; the tripod is clamped and transferred to the patient's eye at operation - two limbs being placed at 3 o'clock and 9 o'clock on the limbus and the third limb indicating the mark in the sclera where the position of the hole is estimated. I find it difficult to believe that this somewhat complicated method will give better results than our plan of marking at the limbus, with Indian ink, under direct vision with an electric ophthalmoscope, the meridian 
In which the hole is situated, and estimating in disc diameters the distance of the hole in that meridian from the most anterior part of the fundus visible. Having marked on the sclera the estimated position of the hole, Weve proceeds to puncture the sclera and choroid and to reach the retinal hole, using a fine blunt needle electrode and a uni-polar diathermy current of 40 to 50 milliampères (the indifferent electrode is not employed); the needle first engages the sclera, the current is then turned on when, with the minimum of pressure, it sinks into the eye. An ophthalmoscopic examination is now made; to facilitate this, as the cornea so readily becomes dry under cocaine, he keeps a corneal contact lens $(11 \mathrm{~mm}$. diameter and $7.5 \mathrm{~mm}$. radius of curvature) in position throughout the operation; this is readily adjusted before each examination. A white, fluffy, very obvious area of coagulation is seen and its position in relation to the hole noted as a guide to further punctures. A series of punctures is made in this way until the entire margin of the tear is coagulated, a fundus examination being made after each. Sub-retinal fluid is only lost gradually throughout the operation but it must be ensured, finally, that sufficient has been evacuated to bring the retina approximately in contact with the choroid. This operation is beautiful in its precision and it is remarkable the ease and certainty with which a retinal hole can be closed under direct vision. It would, however, seem to share with Gonin's operation the objection of plunging the cautery into the vitreous itself, surely an undesirable practice in any case of detachment, though the diathermy current is obviously a more readily controlled method of producing coagulation than the electro-cautery.

I understand that for cases of oral tear, or so-called anterior retinal dialysis, Weve used a method of superficial diathermy cauterization of the choroid without puncture similar to that employed in the cases to be described.

The second method of treatment by diathermy current, and the one which was used solely in this series of cases, was described by Larsson of Stockholm. It is analogous to the Guist operation in that it aims at producing a diffuse area of choroiditis without any active interference within the vitreous. It is claimed that, if this area of induced choroidal inflammation is so placed that when the sub-retinal fluid is evacuated the retinal hole lies upon it, the communication between the pre-retinal and sub-retinal spaces will be closed and the detachment cured. The only essential difference being that Guist, of course, uses caustic potash to produce the choroidal reaction while here the diathermy current is employed to produce a similar effect.

Whilst acknowledging the fundamental importance of Gonin's observation with, regard to the retinal tear, Larsson considers that 
the hole is usually surrounded by an area of retina which is thin and of lowered resistence, predisposing to the formation of new holes, an observation which can be supported by an examination of the neighbourhood of the hole in most detachments. He suggests that to obtain a cure, adhesion of the retina to the choroid must be obtained not only at the margin of any hole or holes but for a considerable area around. For this reason he considers that accurate localization of the hole no longer has the same value, though, obviously, discovery of a hole is still of prime importance to indicate the site of operation. We have continued to mark out the hole under direct ophthalmoscopy, as for cautery puncture, for without it at operation the globe is liable to become twisted and thereby accurate bearings lost.

I understand that Safar in Vienna is using a small needle electrode carrying a diathermy current, which perforates the sclera and engages in the choroid, producing there localized foci of inflammation. It is maintained that this method is more deliberate than the diffuse cauterization and is less likely to cause choroidal infarction, a complication which has not so far been met with at Moorfields.

\section{The Operation}

Very little discomfort is experienced and no general anaesthetic or narcotic is usually necessary, though hyoscine and morphine or nembutal may be used. The machine used is a standard diathermy apparatus supplied by Messrs. Schall. There are technical difficulties in measuring a diathermy current rapidly, for the ampèremeter used for the currents employed in general diathermy work is dependent on the expansion of a wire to varying temperatures and takes an appreciable time to adjust. Further, such a measurement, while satisfactory for reading in ampères, is of less value for the small currents-up to 100 milliampèreswhich we employ.

I undersand that it is possible to obtain a milliampèremeter which registers more rapidly, dependent on a secondary current generated at a thermo-couple in the main circuit, but I have not seen this used. To overcome these difficulties Larsson estimates the current by placing the indifferent and active electrodes in contact before the operation is begun, the current is turned on and regulated so that a reading of 0.75 to 1 ampère is obtained; the indifferent electrode is now bandaged to the leg or arm and the active electrode attached to that used on the eye itself.

Larsson uses as his active electrode a fine, bent wire at the end of which is a ball $0.66 \mathrm{~mm}$. in diameter. Messrs. Weiss 
made this for us but it seemed unnecessarily pliable so we use a platinum wire of the same diameter, 1.5 inches long, bent in the middle at $130^{\circ}$. A vulcanite speculum is useful since if the electrode touches a metal speculum the patient experiences some pain. A sub-conjunctival and sub-Tenon's capsule injection of novocaine and adrenalin is given in the neighbourhood of the hole; a guidestitch, knotted at one end, is passed through the limbus at the ink mark diametrically opposite the hole. A flap of conjunctiva and Tenon's capsule is dissected up and the edges of the muscles isolated in that quadrant of the globe where the hole is situated; the guide-stitch is threaded through Desmarre's retractor and drawn taut so that it crosses the centre of the cornea. Along this guide the estimated distance of the hole from the limbus is measured off with calipers (allowing $8 \mathrm{~mm}$. in an emmetrope and $9 \mathrm{~mm}$. in a myope as the distance from the limbus to the most anterior portion of the fundus visible and $1.5 \mathrm{~mm}$. for each disc diameter of measurement in the fundus). The surface marking of the hole having been ascertained it is marked on the sclera by one or two turns of a $1.5 \mathrm{~mm}$. trephine; this spot is to be the centre of the cauterized area. It is important to remove all episcleral tissue and to get the field of operation quite dry; bleeding from fine vessels can be stopped readily by touching them with a small metal rod, e.g., a lacrymal probe, heated almost to red heat in a spirit flame. To obtain good access to the field of operation a traction stitch through the sclera just outside the limbus is useful. Having again identified the groove made by the trephine, which indicates the position of the hole, cauterization is commenced at this spot. The electrode must be in contact with the sclera before the current is turned on ; five seconds is allowed and the current is then turned off before the electrode is removed. The required reaction is soon learnt; there is an immediate flattening and drying of the sclera in the neighbourhood of the electrode and a greenish, brown ring appears around it. It is well to start with a low current $(0.75$ amperre with the active and indifferent electrodes in contact) and to increase it gradually, if necessary, for it is quite easy, if too strong a current is employed, to perforate the sclera and choroid at the first application ; further, the reaction to a given current varies with each individual patient. This is repeated over the required area of the sclera, about $1.5 \mathrm{~mm}$. interval being allowed between each application. The distribution of the cauterization will depend on the size and shape of the hole and should cover widely the estimated margins of the hole and any thin areas of the retina which were detected. An oral tear or anterior retinal dialysis will, of course, only require a curved area of cauterization starting and ending in the ciliary region and encircling the tear. The presence of the ciliary body at $8-9 \mathrm{~mm}$ 
from the limbus must always be kept in mind and excessive cauterization in this area avoided. Muscles can usually be pulled aside, though if this does not give sufficient access they should be detached and re-inserted at the end of the operation. Separate holes in different regions of the globe can, of course, be treated at the same operation. In cases where no hole is visible a band of cauterization, some $2 \mathrm{~mm}$. wide, at a distance of about $11 \mathrm{~mm}$. from the limbus, may be applied in each quadrant of the globe where detachment exists; this is done on the assumption that a hole is present but is too far forward to be seen with the ophthalmoscope; however, the prognosis in these cases seems very poor. Finally the sub-retinal fluid is evacuated in the region where the detachment is deepest; this may be done by allowing the electrode to stay in contact with the sclera at one spot or by trephining the sclera and then perforating the choroid with a blunt instrument. Larsson favours the latter as he thinks thereby drainage of the sub-retinal fluid may continue for some while but it hardly seems probable that a small perforation in the choroid will remain patent for long. Finally, any detached muscles are re-inserted and the conjunctiva stitched. If the fundus be now examined a diffuse, white area in the choroid is visible and a rough estimate can be made as to its relation to the hole. Both eyes are bandaged and the patient nursed in a position which allows the hole to be dependent, though we have not thought it necessary to raise the foot of the bed in cases where the hole is in the upper part of the fundus. Atropine is instilled daily from the first and a preliminary examination of the fundus made after 48 hours, provided the excursions of the eye are limited. In favourable cases the detachment will be much shallower, though a considerable amount often remains for a few days. If, after five or six days, gross detachment still persists, the operation is unsuccessful and the patient should be allowed up. In successful cases, any detachment which is present at first rapidly settles down, though considerable swelling of the coagulated choroid, over which, of course, the retina is raised, is usual and this is often quite obvious for two or three weeks. After two weeks the patient is allowed up with both eyes uncovered; glasses in which only a small central aperture is present, designed to ensure movements of the head rather than of the eyes, can be prescribed, but it seems that, after a fortnight, favourable cases may be allowed reasonable activities with safety.

Of the 31 cases 18 were cured, i.e., 58.06 per cent. Eleven of these left hospital with no detachment whatever visible; in seven there was seen, around the cauterized area, a certain amount of residual detachment due, no doubt, to local swelling of, and possibly exudate from, the choroid. In no case has an increase 
of this local detachment been noted at subsequent examinations, so I have thought it fair to include them as cures. In most cured cases the field to a one degree object at one-third of a metre was full, or showed only a small loss corresponding to the operation site, and it was often very remarkable how little field defect was produced by extensive choroidal scarring, though no doubt this would have been more obvious if a small object on the screen had been used. The improvement in central vision was variable; if the macula had been detached, from counting fingers vision of $6 / 60$ or $3 / 36$ was usually obtained, while in those cases where the macula was still attached, an increase of one or two lines on Snellen's type was general.

One cured case is of sufficient interest to merit special mention. He was first admitted with an intra-ocular foreign body which could be seen lying in the retina down and out. The lens was not injured and the vision was $6 / 18$. The foreign body was successfully removed by the anterior route and his convalescence was uneventful. He continued to attend the out-patient department regularly and after six weeks complained that the vision of the eye was failing; examination showed an extensive detachment below with corresponding field defect and a small hole at the original site of the foreign body. The macula was not involved and the vision 6/36. Diathermy was applied to the lower and outer segment of the eye; the retina went back completely, and he was discharged with a full field and 6/24 vision.

In the series no sort of selection was made. The average age of the patient and duration of symptoms were 43 years and 33 weeks respectively in cases where the operation failed, and 41 years and 6 weeks respectively in those cases cured. Further, in seven of the cases one or more previous operations, either Gonin's or Guist's, had been done, and in only one of these was the result successful. In 6 of the 31 cases no hole was found and, again, in only one was the result satisfactory. Obviously if the more unfavourable cases bc rejected the figure of 58.06 per cent. of cures can be improved.

Larsson in 40 unselected cases had 50 per cent. of cures, and in $3 ?$ selected cases 60 per cent. of cures: Complications were notably absent following the operation. A fair degree of local external reaction was usual but soon settled down. In no case was there any marked increase of vitreous opacities attributable to the operation, nor was there any large vitreous haemorrhage, though sub-retinal and intra-choroidal extravasations of blood were seen. Thrombosis of a vortex vein did not occur. I understand that in Vienna embolism of a choroidal artery leading to an area of choroidal atrophy, which may spread to the macular area, has been observed following diathermy, but we did not experience this. 


\title{
Conclusions
}

Whilst this series of cases is too small to allow of any final opinion I think it may fairly justify the following conclusions :

1. That in diathermy we have a method of cauterization for the treatment of retinal detachment preferable to the thermocautery used by Gonin or the solid caustic potash used by Guist. Further, the technical difficulties are considerably less than in the latter operation.

2. Cases where no hole can be found and those in which a previous operation for detachment, of any type, has been done, offer small hope of success.

3 . The age of the patient is of small importance but the prognosis is much less favourable when the symptoms of detachment are of long standing.

4. Finally, as might be expected, almost all cases showing inflammatory signs, post-cortical lens changes, gross vitreous opacities, and hypotension do badly, while those with a relatively clear vitreous, discrete hole or holes, and a normal tension, are the most favourable.

A new diathermy apparatus has now been installed at Moorfields to replace that used in this series of cases. This machine, which was supplied by Messrs. John Weiss \& Co., has been specially made for this operation. It generates a diathermy current of low frequency and has a milliampèremeter which gives rapid readings up to 100 milliampères. I have had no practical experience with it but I understand that, using a current of approximately 60 milliampères, results have been very encouraging.

I would like to express to the Honorary Staff of Moorfields Eye Hospital my gratitude for their permission to publish these cases.

THE HISTOLOGICAL APPEARANCES OF AN EYE SUCCESSFULLY TREATED BY DIATHERMY FOR RETINAL DETACHMENT. FATAL TERMINATION FROM PULMONARY THROMBOSIS ON THE NINETEENTH DAY AFTER OPERATION

BY

\author{
H. B. Stallard \\ LONDON
}

THE opportunities for histological examination of human eyes subjected to the surgical treatment of retinal detachment are rare. Gonin's cauterization of retinal tears has been succeeded by Guist's multiple trephines and caustic applications and now Larsson's 